\title{
On Weak Solutions to the Linear Boltzmann Equation with Inelastic Coulomb Collisions
}

\author{
Rolf Pettersson \\ Department of Mathematics, Chalmers University of Technology, SE-412 96 Göteborg, Sweden
}

\begin{abstract}
This paper considers the time- and space-dependent linear Boltzmann equation with general boundary conditions in the case of inelastic (granular) collisions. First, in the (angular) cut-off case, mild $L^{1}$-solutions are constructed as limits of the iterate functions and boundedness of higher velocity moments are discussed in the case of inverse power collisions forces. Then the problem of the weak solutions, as weak limit of a sequence of mild solutions, is studied for a bounded body, in the case of very soft interactions (including the Coulomb case). Furthermore, strong convergence of weak solutions to the equilibrium, when time goes to infinity, is discussed, using a generalized H-theorem, together with a translation continuity property.
\end{abstract}

Keywords: linear Boltzmann equation, weak solutions, granular collisions, Coulomb interactions.

PACS: $02.30 . \mathrm{Rz}, 05.20 . \mathrm{Dd}, 45.70 .-\mathrm{n}, 47.45 . \mathrm{Ab}, 51.10 .+\mathrm{y}$.

\section{INTRODUCTION}

The linear Boltzmann equation is frequently used for mathematical modelling in physics, (e.g. for describing the neutron distribution in reactor physics, cf. [1]-[3]). In our earlier papers [4]-[6] we have studied the linear Boltzmann equation, both in the angular cut-off case and the infinite range case, for a function $f(\mathbf{x}, \mathbf{v}, t)$ representing the distribution of particles with mass $m$ undergoing elastic binary collision with other particles with mass $m_{*}$ and with a given (known) distribution function $Y\left(\mathbf{x}, \mathbf{v}_{*}\right)$. In recent years there has been a significant interest in the study of kinetic models for granular flows, mostly with the non-linear Boltzmann equation; see ref. [7] for an overview, with many further references, and also [8]-[9]. Our papers [10] and [11] consider respectively the time-dependent and the stationary linear Boltzmann equation for inelastic (granular) collisions, both papers in the angular cut-off case, but the paper [12] studies the (granular) infinite range case.

The purpose of this paper is to generalize our earlier results in [13] on existence of weak solutions for very soft elastic collisions (including the Coulomb forces), in the Cauchy problem case, to inelastic (granular) collisions in a bounded body, and (for simplicity) without external forces.

So we will study collisions between particles with mass $m$ and particles with mass $m_{*}$, such that momentum is conserved, $m \mathbf{v}+m_{*} \mathbf{v}_{*}=m \mathbf{v}^{\prime}+m_{*} \mathbf{v}_{*}^{\prime}$, where $\mathbf{v}, \mathbf{v}_{*}$ are velocities before and $\mathbf{v}^{\prime}, \mathbf{v}_{*}^{\prime}$ are velocities after a collision.

In the elastic case, where also kinetic energy is conserved, one finds that the velocities after a binary collision terminate on two concentric spheres, so all velocities $\mathbf{v}^{\prime}$ lie on a sphere with radius $\frac{m_{*} w}{m+m_{*}}$ around the center of mass, $\overline{\mathbf{v}}=\left(m \mathbf{v}+m_{*} \mathbf{v}_{*}\right) /\left(m+m_{*}\right)$, where $w=\left|\mathbf{v}-\mathbf{v}_{*}\right|$, and all velocities $\mathbf{v}_{*}^{\prime}$ lie on a sphere with the same center $\overline{\mathbf{v}}$ and with radius $\frac{m w}{m+m_{*}}$, cf Figure 1 in [4].

In the granular, inelastic case we assume the following relation between the relative velocity components normal to the plane of contact of the two particles,

$$
\mathbf{w}^{\prime} \cdot \mathbf{u}=-a(\mathbf{w} \cdot \mathbf{u}),
$$

where $a$ is a constant, $0<a \leq 1$, and $\mathbf{w}=\mathbf{v}-\mathbf{v}_{*}, \mathbf{w}^{\prime}=\mathbf{v}^{\prime}-\mathbf{v}_{*}^{\prime}$ are the relative velocities before and after the collision, and $\mathbf{u}$ is a unit vector in the direction of impact, $\mathbf{u}=\left(\mathbf{v}-\mathbf{v}^{\prime}\right) /\left|\mathbf{v}-\mathbf{v}^{\prime}\right|$. Then we find that $\mathbf{v}^{\prime}=\mathbf{v}_{a}^{\prime}$ lies on the line between $\mathbf{v}$ and $\mathbf{v}_{1}^{\prime}$, where $\mathbf{v}_{1}^{\prime}$ is the postvelocity in the case of elastic collision, i.e. with $a=1$, and $\mathbf{v}_{* a}^{\prime}$ lies on the (parallel) line between $\mathbf{v}_{*}$ and $\mathbf{v}_{* 1}^{\prime}$.

Now the following relations hold for the velocities in a granular, inelastic collision

$$
\mathbf{v}^{\prime}=\mathbf{v}-(a+1) \frac{m_{*}}{m+m_{*}}(\mathbf{w} \cdot \mathbf{u}) \mathbf{u}, \quad \mathbf{v}_{*}^{\prime}=\mathbf{v}_{*}+(a+1) \frac{m}{m+m_{*}}(\mathbf{w} \cdot \mathbf{u}) \mathbf{u},
$$

where $\mathbf{w} \cdot \mathbf{u}=w \cos \theta, w=\left|\mathbf{v}-\mathbf{v}_{*}\right|$, if the unit vector $\mathbf{u}$ is given in spherical coordinates,

$$
\mathbf{u}=(\sin \theta \cos \phi, \sin \theta \sin \phi, \cos \theta), 0 \leq \theta \leq \pi / 2,0 \leq \phi<2 \pi .
$$

27th International Symposium on Rarefied Gas Dynamics, 2010 
Moreover, if we change notations, and let ${ }^{\prime} \mathbf{v}, \mathbf{v}_{*}$ be the velocities before, and $\mathbf{v}, \mathbf{v}_{*}$ the velocities after a binary inelastic collision, then by (1) and (2), cf. [7]-[12],

$$
' \mathbf{v}=\mathbf{v}-\frac{(a+1) m_{*}}{a\left(m+m_{*}\right)}(\mathbf{w} \cdot \mathbf{u}) \mathbf{u}, \quad \mathbf{v}_{*}=\mathbf{v}_{*}+\frac{(a+1) m}{a\left(m+m_{*}\right)}(\mathbf{w} \cdot \mathbf{u}) \mathbf{u} .
$$

\section{PRELIMINARIES}

We consider the time-dependent transport equation for a distribution function $f(\mathbf{x}, \mathbf{v}, t)$, depending on a space variable $\mathbf{x}=\left(x_{1}, x_{2}, x_{3}\right)$ in a bounded convex body $D$ with (piecewise) $C^{1}$-boundary $\Gamma=\partial D$, and depending on a velocity variable $\mathbf{v}=\left(v_{1}, v_{2}, v_{3}\right) \in V=\mathbb{R}^{3}$ and a time variable $t \in \mathbb{R}_{+}$. Then the linear Boltzmann equation is in the strong form

$$
\begin{array}{r}
\frac{\partial f}{\partial t}(\mathbf{x}, \mathbf{v}, t)+\mathbf{v} \operatorname{grad}_{\mathbf{x}} f(\mathbf{x}, \mathbf{v}, t)=(Q f)(\mathbf{x}, \mathbf{v}, t), \\
\mathbf{x} \in D, \mathbf{v} \in V=\mathbb{R}^{3}, t \in \mathbb{R}_{+},
\end{array}
$$

supplemented by initial data

$$
f(\mathbf{x}, \mathbf{v}, 0)=f_{0}(\mathbf{x}, \mathbf{v}), \quad \mathbf{x} \in D, \mathbf{v} \in V .
$$

The collision term can, in the case of inelastic (granular) collision, be written, cf. [7]-[12],

$$
(Q f)(\mathbf{x}, \mathbf{v}, t)=\int_{V} \int_{\Omega}\left[J_{a}(\theta, w) Y\left(\mathbf{x}, \mathbf{v}_{*}\right) f\left(\mathbf{x}, \mathbf{v}^{\prime}, t\right)-Y\left(\mathbf{x}, \mathbf{v}_{*}\right) f(\mathbf{x}, \mathbf{v}, t)\right] B(\theta, w) d \mathbf{v}_{*} d \theta d \phi
$$

with $w=\left|\mathbf{v}-\mathbf{v}_{*}\right|$, where $Y \geq 0$ is a known distribution, $B \geq 0$ is given by the collision process, and finally $J_{a}$ is a factor depending on the granular process (and giving mass conservation, if the gain and the loss integrals converge separately). Furthermore, ${ }^{\prime} \mathbf{v},{ }^{\prime} \mathbf{v}_{*}$ in (7) are the velocities before and $\mathbf{v}, \mathbf{v}_{*}$ the velocities after the binary collision, cf. (4), and $\Omega=\{(\theta, \phi): 0 \leq \theta<\hat{\theta}, 0 \leq \phi<2 \pi\}$ represents the impact plane, where $\hat{\theta}<\frac{\pi}{2}$ in the angular cut-off case,

and $\hat{\theta}=\frac{\pi}{2}$ in the infinite range case. The collision function $B(\theta, w)$ is in the physically interesting case with inverse $\mathrm{k}$-th power collision forces given by

$$
B(\theta, w)=b(\theta) w^{\gamma}, \quad \gamma=\frac{k-5}{k-1}, w=\left|\mathbf{v}-\mathbf{v}_{*}\right|,
$$

with hard forces for $k>5$, Maxwellian for $k=5$, and soft forces for $3<k<5$, where $b(\theta)$ has a non-integrable singularity for $\theta=\frac{\pi}{2}$, of order $\alpha=-(k+1) /(k-1)$. So in the angular cut-off case one can choose $\hat{\theta}<\frac{\pi}{2}$, and then the gain and the loss terms can be separated $(Q f)(\mathbf{x}, \mathbf{v}, t)=\left(Q^{+} f\right)(\mathbf{x}, \mathbf{v}, t)-\left(Q^{-} f\right)(\mathbf{x}, \mathbf{v}, t)$, where the gain term can be written (with a kernel $K_{a}$ )

$$
\left(Q^{+} f\right)(\mathbf{x}, \mathbf{v}, t)=\int_{V} K_{a}(\mathbf{x}, ' \mathbf{v} \rightarrow \mathbf{v}) f\left(\mathbf{x}^{\prime}, \mathbf{v}, t\right) d^{\prime} \mathbf{v},
$$

and the loss term is written with the collision frequency $L(\mathbf{x}, \mathbf{v})$ as $\left(Q^{-} f\right)(\mathbf{x}, \mathbf{v}, t)=L(\mathbf{x}, \mathbf{v}) f(\mathbf{x}, \mathbf{v}, t)$. In the case of non-absorbing body we have that $L(\mathbf{x}, \mathbf{v})=\int_{V} K_{a}\left(\mathbf{x}, \mathbf{v} \rightarrow \mathbf{v}^{\prime}\right) d \mathbf{v}^{\prime}$. Furthermore, equation (5) is in the space-dependent case supplemented by ( general) boundary conditions

$$
\begin{aligned}
f_{-}(\mathbf{x}, \mathbf{v}, t)=\int & \frac{|\mathbf{n} \cdot \tilde{\mathbf{v}}|}{|\mathbf{n} \cdot \mathbf{v}|} R(\mathbf{x}, \tilde{\mathbf{v}} \rightarrow \mathbf{v}) f_{+}(\mathbf{x}, \tilde{\mathbf{v}}, t) d \tilde{\mathbf{v}}, \\
& \mathbf{n} \cdot \mathbf{v}<0, \mathbf{n} \cdot \tilde{\mathbf{v}}>0, \mathbf{x} \in \Gamma=\partial D, t \in \mathbb{R}_{+},
\end{aligned}
$$

where $\mathbf{n}=\mathbf{n}(\mathbf{x})$ is the unit outward normal at $\mathbf{x} \in \Gamma=\partial D$. The function $R \geq 0$ satisfies (in the non-absorbing boundary case) $\int_{V} R(\mathbf{x}, \tilde{\mathbf{v}} \rightarrow \mathbf{v}) d \mathbf{v} \equiv 1$, and $f_{-}$and $f_{+}$represent the ingoing and outgoing trace functions corresponding to $f$. In the specular reflection case the function $R$ is represented by a Dirac measure $R(\mathbf{x}, \tilde{\mathbf{v}} \rightarrow \mathbf{v})=\delta(\mathbf{v}-\tilde{\mathbf{v}}+2(\mathbf{n} \cdot \tilde{\mathbf{v}}) \mathbf{n})$, and in the diffuse reflection case $R(\mathbf{x}, \tilde{\mathbf{v}} \rightarrow \mathbf{v})=|\mathbf{n} \cdot \mathbf{v}| W(\mathbf{x}, \mathbf{v})$ with some given function $W \geq 0$, (e.g. Maxwellian function).

Let $t_{b} \equiv t_{b}(\mathbf{x}, \mathbf{v})=\inf _{\tau \in \mathbb{R}_{+}}\{\tau: \mathbf{x}-\tau \mathbf{v} \notin D\}$, and $\mathbf{x}_{b} \equiv \mathbf{x}_{b}(\mathbf{x}, \mathbf{v})=\mathbf{x}-t_{b} \mathbf{v}$, where $t_{b}$ represents the time for a particle going with velocity $\mathbf{v}$ from the boundary point $\mathbf{x}_{b}$ to the point $\mathbf{x}$.

Then, using differentiation along the characteristics, equation (5) can formally be transformed to a mild equation, and also to an exponential form of equation in the angular cut-off case, cf. [10] and also [4]-[6]. 


\section{CONSTRUCTION OF SOLUTIONS IN THE CUT-OFF CASE}

We construct $L^{1}$-solutions to our problems as limits of iterate functions $f^{n}$, when $n \rightarrow \infty$. Let first $f^{-1}(\mathbf{x}, \mathbf{v}, t) \equiv 0$. Then define for given $f^{n-1}$ the next iterate $f^{n}$, first at the ingoing boundary (using the appropriate boundary condition), and then inside $D$ and at the outgoing boundary (using the exponential form of the equation),

$$
\begin{gathered}
f_{-}^{n}(\mathbf{x}, \mathbf{v}, t)=\int_{V} \frac{|\mathbf{n} \cdot \tilde{\mathbf{v}}|}{|\mathbf{n} \cdot \mathbf{v}|} R(\mathbf{x}, \tilde{\mathbf{v}} \rightarrow \mathbf{v}) f_{+}^{n-1}(\mathbf{x}, \tilde{\mathbf{v}}, t) d \tilde{\mathbf{v}} \\
f^{n}(\mathbf{x}, \mathbf{v}, t)=\bar{f}^{n}(\mathbf{x}, \mathbf{v}, t) \exp \left[-\int_{0}^{t} L(\mathbf{x}-s \mathbf{v}, \mathbf{v}) d s\right]+ \\
+\int_{0}^{t} \exp \left[-\int_{0}^{\tau} L(\mathbf{x}-s \mathbf{v}, \mathbf{v}) d s\right] \int_{V} K_{a}\left(\mathbf{x}-\tau \mathbf{v},{ }^{\prime} \mathbf{v} \rightarrow \mathbf{v}\right) f^{n-1}\left(\mathbf{x}-\tau \mathbf{v},,^{\prime} \mathbf{v}, t-\tau\right) d^{\prime} \mathbf{v} d \tau,
\end{gathered}
$$

where

$$
\bar{f}^{n}(\mathbf{x}, \mathbf{v}, t)=\left\{\begin{array}{lr}
f_{0}(\mathbf{x}-t \mathbf{v}, \mathbf{v}), & 0 \leq t \leq t_{b}, \\
f_{-}^{n}\left(\mathbf{x}_{b}, \mathbf{v}, t-t_{b}\right), & t>t_{b}
\end{array}\right.
$$

Let also $f^{n}(\mathbf{x}, \mathbf{v}, t) \equiv 0$ for $\mathbf{x} \in \mathbb{R}^{3} \backslash D$. Now we get a monotonicity lemma, $f^{n}(\mathbf{x}, \mathbf{v}, t) \geq f^{n-1}(\mathbf{x}, \mathbf{v}, t)$, which is essential and can be proved by induction.

Then, by differentiation along the characteristics and integration (with Green's formula), we find (using the equations above, cf. [10]), that

$$
\int_{D} \int_{V} f^{n}(\mathbf{x}, \mathbf{v}, t) d \mathbf{x} d \mathbf{v} \leq \int_{D} \int_{V} f_{0}(\mathbf{x}, \mathbf{v}) d \mathbf{x} d \mathbf{v}
$$

so Levi's theorem (on monotone convergence) gives existence of (mild) $L^{1}$-solutions

$$
f(\mathbf{x}, \mathbf{v}, t)=\lim _{n \rightarrow \infty} f^{n}(\mathbf{x}, \mathbf{v}, t)
$$

to our problem with granular gases (almost in the same way as for the elastic collision case). Furthermore, if $L(\mathbf{x}, \mathbf{v}) f(\mathbf{x}, \mathbf{v}, t) \in L^{1}(D \times V)$, then we get equality in (14) for the limit function $f$, giving mass conservation together with uniqueness in the relevant function space (cf [4]-[6], [10], [11], and also Proposition 3.3, chapter 11 in [3]).

Remark 1 The assumption $L f \in L^{1}(D \times V)$ is, for instance, satisfied for the solution $f$ in the case of inverse power collision forces, cf. (8), together with e.g. specular boundary reflections. This follows from a statement on global boundedness (in time) of higher velocity moments, (cf. Theorem 4.1 and Corollary 4.1 in [10]).

Remark 2 There holds also in the granular inelastic collision case an $\mathrm{H}$-theorem for a general relative entropy functional

$$
H_{F}^{\Phi}(f)(t)=\int_{D} \int_{V} \Phi\left(\frac{f(\mathbf{x}, \mathbf{v}, t)}{F(\mathbf{x}, \mathbf{v})}\right) F(\mathbf{x}, \mathbf{v}) d \mathbf{x} d \mathbf{v},
$$

giving that this H-functional is nonincreasing in time, if $\Phi=\Phi(z), \mathbb{R}_{+} \rightarrow \mathbb{R}$, is a convex $C^{1}$-function, and if there exists a corresponding stationary solution $F(\mathbf{x}, \mathbf{v})$ with the same total mass as the initial data $f_{0}(\mathbf{x}, \mathbf{v})$ for the time-dependent solution $f(\mathbf{x}, \mathbf{v}, t)$; cf. Theorem 5.1 in [10]. By using this $\mathrm{H}$-functional one can prove that every time-dependent solution $f(\mathbf{x}, \mathbf{v}, t)$ converges to the corresponding stationary solution $F(\mathbf{x}, \mathbf{v})$, as time goes to infinity; cf. Remark 5.1 in [10] and further references.

\section{WEAK SOLUTIONS IN THE COULOMB CASE}

In this section the linear Boltzmann equation for granular inelastic collisions is considered with a weak form of angular cut-off, (cf. (16) below), in the collision term for very soft forces, i.e. with $9 / 5<k \leq 3$, so $-4<\gamma \leq-1$ in equation (8). We will here (for simplicity) consider the Coulomb forces, i.e. with $k=2$ and $\gamma=-3$ in (8). The problem 
is studied in the following weak integral form, which can formally be derived from equation (5) with (6) and (7):

$$
\begin{aligned}
\int_{D} \int_{V} g & (\mathbf{x}, \mathbf{v}, t) f(\mathbf{x}, \mathbf{v}, t) d \mathbf{x} d \mathbf{v}=\int_{D} \int_{V} g(\mathbf{x}, \mathbf{v}, 0) f_{0}(\mathbf{x}, \mathbf{v}) d \mathbf{x} d \mathbf{v}+ \\
& \int_{0}^{t} \int_{D} \int_{V}\left[\mathbf{v} \cdot \operatorname{grad}_{\mathbf{x}} g(\mathbf{x}, \mathbf{v}, \tau)+\frac{\partial}{\partial \tau} g(\mathbf{x}, \mathbf{v}, \tau)\right] f(\mathbf{x}, \mathbf{v}, \tau) d \mathbf{x} d \mathbf{v} d \tau+ \\
& \int_{0}^{t} \int_{D} \int_{V} \int_{V} \int_{\Omega}\left[g\left(\mathbf{x}, \mathbf{v}^{\prime}, \tau\right)-g(\mathbf{x}, \mathbf{v}, \tau)\right] B(\theta, w) Y\left(\mathbf{x}, \mathbf{v}_{*}\right) f(\mathbf{x}, \mathbf{v}, \tau) d \mathbf{x} d \mathbf{v} d \mathbf{v}_{*} d \theta d \phi d \tau,
\end{aligned}
$$

for all test functions $g \in C_{0 ; \sigma}^{1, \infty}=\left\{g \in C_{\sigma}^{1, \infty}: g(\mathbf{x}, \mathbf{v}, t)=0, \mathbf{x} \in \Gamma=\partial D\right\}$, which (for simplicity) are zero on the boundary. Here $C_{\sigma}^{1, \infty}=\left\{g \in C^{1}\left(D \times V \times \mathbb{R}_{+}\right):\|g\|_{\sigma}<\infty\right\}$, where

$$
\begin{aligned}
\|g\|_{\sigma} & =\sup \left[\left(1+v^{2}\right)^{-\sigma / 2}|g(\mathbf{x}, \mathbf{v}, t)|\right]+\sup \left[\left(1+v^{2}\right)^{-\sigma / 2}\left|\frac{\partial}{\partial \tau} g(\mathbf{x}, \mathbf{v}, t)\right|\right] \\
& +\sup \left[\left(1+v^{2}\right)^{-(\sigma-1) / 2}\left|\nabla_{\mathbf{x}} g(\mathbf{x}, \mathbf{v}, t)\right|\right]+\sup \left[\left(1+v^{2}\right)^{-(\sigma-1) / 2}\left|\nabla_{\mathbf{v}} g(\mathbf{x}, \mathbf{v}, t)\right|\right],
\end{aligned}
$$

(giving a wider class of test functions than used in a similar context, cf. e.g. [12]).

We start our calculations with a lemma, estimating the difference between the test functions in the collision term(16); cf. Lemma 2.3. in [13].

Lemma 1. Let $g=g(\mathbf{v})$ be a continuously differentiable function. Then for $\sigma \geq 0$ there holds that $\left|g\left(\mathbf{v}^{\prime}(\theta)\right)-g(\mathbf{v})\right| \leq$ const $\times \sup \left[\left(1+u^{2}\right)^{-\sigma / 2} \mid \nabla g(\mathbf{u} \mid] \cdot w \cos \theta\left(1+v^{2}\right)^{\sigma / 2}\left(1+v_{*}^{2}\right)^{\sigma / 2}\right.$.

Proof. $\left|g\left(\mathbf{v}^{\prime}(\theta)\right)-g(\mathbf{v})\right|=\left|\int_{\theta}^{\pi / 2} \nabla g\left(\mathbf{v}^{\prime}(\psi)\right) \frac{\partial}{\partial \psi} \mathbf{v}^{\prime}(\psi) d \psi\right| \leq \sup _{\mathbf{u}}\left[\left(1+u^{2}\right)^{-\sigma / 2}|\nabla g(\mathbf{u})|\right] \int_{\theta}^{\pi / 2}\left(1+\left(\mathbf{v}^{\prime}(\psi)\right)^{2}\right)^{\sigma / 2}\left|\frac{\partial}{\partial \psi} \mathbf{v}^{\prime}(\psi)\right| d \psi$, where (2) (in the granular case) gives $\left|\frac{\partial}{\partial \psi} \mathbf{v}^{\prime}(\psi)\right|=\frac{(a+1) m}{\left(m+m_{*}\right)} w, w=\left|\mathbf{v}-\mathbf{v}_{*}\right|$, and $1+\left(\mathbf{v}^{\prime}\right)^{2} \leq \max \left(1, \frac{m_{*}}{m}\right) \cdot\left(1+v^{2}\right)\left(1+v_{*}^{2}\right)$, so the lemma follows.

Next we will formulate a proposition on estimates of velocities after and before a binary (elastic or inelastic) collision; cf. also ref. [4], where the negative term (on right hand side below) is used to get boundedness in time of higher velocity moments.

Proposition 2. Let $\mathbf{v}_{a}^{\prime}(\theta, \phi)$ and $\mathbf{v}$ be the velocities after and before a collision. Then for all $\sigma>0$ there are positive constants $K_{1}$ and $K_{2}$ (depending on $\sigma, m, m_{*}$, and a) such that

$$
\left(1+\left|\mathbf{v}_{a}^{\prime}(\theta, \phi)\right|^{2}\right)^{\sigma / 2}-\left(1+|\mathbf{v}|^{2}\right)^{\sigma / 2} \leq K_{1}(w \cos \theta)\left(1+v_{*}\right)^{\max (1, \sigma-1)}\left(1+v^{2}\right)^{(\sigma-2) / 2}-K_{2}\left(w \cos ^{2} \theta\right)\left(1+v^{2}\right)^{(\sigma-1) / 2}
$$

Now we will study the case with a sequence of bounded collision functions; $\mathrm{cf}$. (8) for $k=2, \gamma=-3$, in the Coulomb case. Let for positive integers $N$

$$
B_{N}(\theta, w)=\min (N, B(\theta, w))
$$

and let $Q_{N}$ be the corresponding collision term with mild solutions $f_{N}$. Then we can formulate a proposition of existence of the solutions with mass conservation and local boundedness in time of higher velocity moments, for the case with some weak angular cut-off (independent of $N$ ).

Proposition 3. A) Let for the Coulomb case $(k=2, \gamma=-3), B_{N}$ be given by (18), and assume that $\sup \left(\int Y\left(\mathbf{x}, \mathbf{v}_{*}\right) d \mathbf{v}_{*}\right)<\infty$. Then, for every initial function $f_{0} \in L_{+}^{1}(D \times V)$ and general boundary function $R$, there exists (for inelastic or elastic collisions) a unique mild solution $f_{N}$ to the problem (5)-(7) with non-absorbing boundary, giving mass conservation.

$$
\int_{D} \int_{V} f_{N}(\mathbf{x}, \mathbf{v}, t) d \mathbf{x} d \mathbf{v}=\int_{D} \int_{V} f_{0}(\mathbf{x}, \mathbf{v}) d \mathbf{x} d \mathbf{v}, t \in \mathbb{R}_{+}
$$


B) Furthermore, assume that $\int_{0}^{\pi / 2} b(\theta) \cos \theta d \theta=b_{0}<\infty$, and that $\left(1+v_{*}\right)^{\max \left(1, \sigma_{0}-1\right)} \sup _{\mathbf{x}} Y\left(\mathbf{x}, \mathbf{v}_{*}\right) \in L^{q}(V)$ for $3<q \leq \infty$ and some $\sigma_{0}>0$, and suppose a "non-heating" boundary (e.g. specular reflection). Then, if $\left(1+v^{2}\right)^{\sigma / 2} f_{0}(\mathbf{x}, \mathbf{v}) \in$ $L^{1}(D \times V)$ there holds a (local) boundedness of higher velocity moments, $0<\sigma \leq \sigma_{0}, t \in \mathbb{R}_{+}$:

$$
\int_{D} \int_{V}\left(1+v^{2}\right)^{\sigma / 2} f_{N}(\mathbf{x}, \mathbf{v}, t) d \mathbf{x} d \mathbf{v} \leq e^{A_{\sigma} t} \int_{D} \int_{V}\left(1+v^{2}\right)^{\sigma / 2} f_{0}(\mathbf{x}, \mathbf{v}) d \mathbf{x} d \mathbf{v}
$$

where $A_{\sigma}$ is a positive constant depending on $B$ only through $b_{0}$.

Proof. Proposition A follows from the discussion in the section above. To get Proposition B, start with differentiation along the characteristics of iterate functions $f_{N}^{n}$ in (16)-(18), multiply by $\left(1+v^{2}\right)^{\sigma / 2}$ and integrate, with some change of variables, cf. [13]. Then, by the proposition (above) on velocities after a binary collision, using only the positive part (at the right hand side) of the estimate, one get the integrals of type

$$
\begin{aligned}
& \int_{0}^{t} \int_{D} \int_{V} \int_{V} \int_{\Omega} w \cos \theta\left(1+v_{*}\right)^{\max (1, \sigma-1)} B_{N}(\theta, w) Y\left(\mathbf{x}, \mathbf{v}_{*}\right) f_{N}^{n}(\mathbf{x}, \mathbf{v}, \tau) d \mathbf{x} d \mathbf{v} d \mathbf{v}_{*} d \theta d \phi d \tau \\
& \quad \leq 2 \pi b_{0} \int_{0}^{t} \int_{D} \int_{V}\left[\sup _{\mathbf{x}} \int_{V} w^{\gamma+1}\left(1+v_{*}\right)^{\max (1, \sigma-1)} Y\left(\mathbf{x}, \mathbf{v}_{*}\right) d \mathbf{v}_{*}\right] \cdot\left(1+v^{2}\right)^{(\sigma-2) / 2} f_{N}^{n}(\mathbf{x}, \mathbf{v}, \tau) d \mathbf{x} d \mathbf{v} d \tau
\end{aligned}
$$

where $\gamma=-3$ for $k=2$. Thus

$$
\int_{V} w^{-2} G\left(\mathbf{v}_{*}\right) d \mathbf{v}_{*} \leq \int_{w<1} w^{-2} G\left(\mathbf{v}_{*}\right) d \mathbf{v}_{*}+\int_{V} G\left(\mathbf{v}_{*}\right) d \mathbf{v}_{*}
$$

where Hölder inequality (for the first integral on r.h.s.), gives

$$
\int_{V} w^{-2} G\left(\mathbf{v}_{*}\right) d \mathbf{v}_{*} \leq\left(\int_{w<1} w^{-2 q^{\prime}} G\left(\mathbf{v}_{*}\right) d \mathbf{v}_{*}\right)^{1 / q^{\prime}}\left(\int_{V}\left(G\left(\mathbf{v}_{*}\right)\right)^{q} d \mathbf{v}_{*}\right)^{1 / q},
$$

if $3<q<\infty$, and $1 / q+1 / q^{\prime}=1$, i.e. $1<q^{\prime}<3 / 2$. Now, using a Gronwall type estimate, the (local) boundedness of velocity moments are reached for the iterates $f_{N}^{n}$, and finally the monotonicity property $f_{N}^{n} \uparrow f_{n}, n \rightarrow \infty$, gives the result; cf. [13] and further references.

The existence theorem for very soft solutions to the linear Boltzmann equation is based on a compactness lemma, which is analogous to that given by Arkeryd in [14], (cf, also Lemma 4.1 in [5]). The proof is omitted here; see Lemma 2.5 in [13].

Lemma 4. Let $\left\{f_{N}\right\}_{N=1}^{\infty}$ be a sequence of nonnegative integrable functions on $D \times V$, such that for some $\sigma_{0}>0$

$$
\iint\left(1+v^{2}\right)^{\sigma_{0} / 2} f_{N}(\mathbf{x}, \mathbf{v}) d \mathbf{x} d \mathbf{v} \leq C_{\sigma_{0}}
$$

with a constant $C_{\sigma_{0}}$ independent of $N$. Let $E=E(\mathbf{x}, \mathbf{v})$ be a positive measurable function satisfying

$$
\sup _{\mathbf{x}, \mathbf{v}}\left[E(\mathbf{x}, \mathbf{v})\left(1+v^{2}\right)^{-\bar{\sigma} / 2}\right]<\infty
$$

for some $\bar{\sigma}<\sigma_{0}$, and let the $H$-functional in (15) with $\Phi=z \log z, z=f / E$, satisfies an inequality $H_{E} f_{N} \leq C_{E}$ with a constant $C_{E}$ independent of $N$. Then there exists a subsequence $\left\{f_{N_{i}}\right\}_{i=1}^{\infty} \subset\left\{f_{N}\right\}_{N=1}^{\infty}$ converging weakly to a nonnegative integrable function on $D \times V$, such that

$$
\iint g(\mathbf{x}, \mathbf{v}) f_{N_{i}}(\mathbf{x}, \mathbf{v}) d \mathbf{x} d \mathbf{v} \rightarrow \iint g(\mathbf{x}, \mathbf{v}) f(\mathbf{x}, \mathbf{v}) d \mathbf{x} d \mathbf{v}, \quad i \rightarrow \infty
$$

for every measurable $g$ satisfying

$$
\sup _{\mathbf{x}, \mathbf{v}}\left[|g(\mathbf{x}, \mathbf{v})|\left(1+v^{2}\right)^{-\sigma / 2}\right]<\infty
$$

with some $0 \leq \sigma<\sigma_{0}$. 
Then we come to the main result in this section concerning the existence of weak solutions and local boundedness (in time) of higher velocity moments in the case of unbounded scattering factor $B$ in equation (8) with some (angular) cut-off, weaker than the cut-offs needed in the theory of mild solutions to the Boltzmann equation. We will formulate the result for the Coulomb case, i.e. with $k=2$ (and $\gamma=-3$ ).

Theorem 5. Let the assumptions in the Lemma above hold, and suppose that the mild solution $f$ satisfies the Htheorem $\left(H_{E} f\right)(t) \leq H_{E}\left(f_{0}\right)<\infty, t \in \mathbf{R}_{+}$. Then there exists a nonnegative solution function $f=f(\mathbf{x}, \mathbf{v}, t)$ to the linear

Boltzmann equation in the weak form (16) for every test function $g \in C_{0 ; \sigma_{o}}^{1, \infty}$, giving mass conservation and also local boundedness of higher moments.

Proof. (Sketch) See mainly the paper [13]. Define first a sequence of functions by (18) and then use the weak compactness lemma together with boundedness of higher moments. Then prove an equicontinuous property for the sequence

$$
\left\{\iint g(\mathbf{x}, \mathbf{v}, t) f_{N}(\mathbf{x}, \mathbf{v}, t) d \mathbf{x} d \mathbf{v}\right\}_{N=1}^{\infty},
$$

on every time interval $[0, T]$, first for rational times, and then for irrational times. Now prove that the function $f$ satisfies the weak equation (16); for details, see [13].

\section{CONVERGENCE TO EQUILIBRIUM}

For the weak solution in (16) we can prove strong convergence to equilibrium, when time goes to infinity, using a generalized H-theorem, cf.(15), with the function $\Phi(z)=(z-1)^{2}, z=f / E$, if the collision function $Y=Y\left(\mathbf{v}_{*}\right)$ is independent of the space-variable, and $E=E(\mathbf{x}, \mathbf{v})$ is a Maxwellian function. Then we first use a cut-off in the initial function, $f_{0, p}=\min \left(f_{0}, p E\right)$ together with a translation property and the weak convergence theorem for a sequence of functions $f_{N}(\mathbf{x}, \mathbf{v}, t)$; for details, see our paper [13].

Remark The assumption $g(\mathbf{x}, \mathbf{v}, t)=0, \mathbf{x} \in \Gamma=\partial D$, on the test functions can be weakened, and general boundary cases can be studied, e.g. specular reflections, cf. [12].

Final remark Granular inelastic collisions can also be studied using transformation of masses and velocities to the problem of elastic collisions, cf. ref. [15].

\section{REFERENCES}

1. N. Bellomo, A. Palczewski, and G. Toscani,Mathematical topics in Nonlinear Kinetic Theory, World Scientific (1989).

2. C. Cercignani, The Boltzmann Equation and its Applications, Springer-Verlag (1988).

3. W. Greenberg, C. van der Mee, and V. Protopopescu, Boundary Value Problems in Abstract Kinetic Theory, Birkhäuser-Verlag (1987).

4. R. Pettersson, On solutions and higher moments for the linear Boltzmann equation with infinite range forces, IMA J. Appl. Math. 38, pp. 151-166 (1987).

5. R. Pettersson, On solution of the linear Boltzmann equation with general boundary conditions and infinite range forces, J. Stat. Phys., 59, pp. 403-440 (1990).

6. R. Pettersson, On weak and strong convergence to equilibrium for solutions of the linear Boltzmann equation, J. Stat. Phys., 72, pp. 355-380 (1993).

7. C. Villani, Mathematics of granular materials, J. Stat. Phys. 124, pp. 781-822 (2006).

8. J.J. Brey, J.W. Dufty, A. Santos, Dissipative dynamics for hard spheres, J. Stat. Phys., 87, pp. 1051-1068 (1997).

9. J.J. Brey, J.W. Dufty, A. Santos, Kinetic models for granular flow, J. Stat. Phys., 97, pp. 281-322 (1999).

10. R. Pettersson, On solution of the linear Boltzmann equation for granular gases, Transp. Th. Stat. Phys. 33, pp. 527-543 (2004).

11. R. Pettersson, On stationary solution of the linear Boltzmann equation with inelastic granular collisions, A I P, Conf. Proc. 762, RGD24, Bari, pp. 252-257 (2005).

12. R. Pettersson, On solutions to the linear Boltzmann equation with inelastic granular collisions and infinite range forces, A I P, Conf. Proc. 1084, RGD26, pp. 135-139 (2008).

13. F. Chvala and R. Pettersson, Weak solutions of the linear Boltzmann equation with very soft collisions, J. Math. Anal. Appl. 191, pp. 360-379 (1995).

14. L. Arkeryd Intermolecular forces of infinite range and the Boltzmann equation, Arch. Rat. Mech. Anal. 77, pp. 11-21 (1981).

15. A. Santos, J. W. Dufty, Dynamics of hard sphere granular impurity, Phys. Rev. Lett. 97, 058001 (2006). 